\title{
OCULAR MANIFESTATION IN PATIENTS WITH CRANIOFACIAL ANOMALIES - A HOSPITAL BASED STUDY
}

\author{
Nishar Ahamed M11, Jayaram Shetty2, Vijay Pai ${ }^{3}$
}

${ }_{1}^{1}$ Postgraduate, Department of Ophthalmology, K. S. Hedge Medical Academy.

2 Professor and HOD, Department of Ophthalmology, K. S. Hedge Medical Academy.

3Professor, Department of Ophthalmology, K. S. Hedge Medical Academy.

\begin{abstract}
BACKGROUND

Ocular manifestations in Children with Craniofacial Anomalies are an important but easily overlooked part as the patient and his family tends to be more focused on the more visible cosmetic disfigurement. The disfigurement while may be corrected in the hands of a competent surgeon at a later date, more lasting harm may be done to the patient's well-being if simple correctable refractive or other ocular conditions are left too late. This study was done to detect ocular involvement in patients with craniofacial anomalies and also to emphasise importance of routine ophthalmic examination in these patients.
\end{abstract}

\section{MATERIALS AND METHODS}

This was a cross-sectional observational type of study. Study duration was dated from dated from November 2012 to October 2014. All patients who presented to the Department of Ophthalmology of Justice KS Hegde Medical College Hospital, Mangalore with craniofacial anomalies were included in this study. All patients with CFA were included in the study irrespective of age of presentation, gender or history of previous corrective surgery. The patients were evaluated at the time of presenting to the Department of Ophthalmology of Justice KS Hegde Medical College, Mangalore. Duration of this study was dated from November 2012 to October 2014.

\section{RESULTS}

Out of the 151 patients screened, $47.68 \%(n=72)$ of the patients had one or the other forms of ocular anomalies. Patients with craniosynostosis had $100 \%$ ocular involvement $(n=39)$. In patients with clefting syndrome, $29.46 \%$ of the patients had ocular involvement ( $\mathrm{n}=33)$. Refractive error was most common ocular anomaly detected in our study. $49.01 \%$ of the patients in our study had refractive error out of which $22.51 \%$ of the patients were hypermetropes.

\section{CONCLUSION}

Findings in our study suggest that there are numerous ocular associations in patients with craniofacial anomalies. A routine ophthalmic evaluation would help in early diagnosis of these ocular conditions and early management which would greatly benefit the patient.

\section{KEYWORDS}

Craniofacial Anomalies, Craniosynostosis, Clefting Syndromes, Hypermetropia.

HOW TO CITE THIS ARTICLE: Ahamed MN, Shetty J, Pai V.Ocular manifestation in patients with craniofacial anomalies-a hospital based study. J. Evolution Med. Dent. Sci. 2016;5(91):6745-6749, DOI: 10.14260/Jemds/2016/1524

\section{BACKGROUND}

Congenital anomalies (CA) - Out of all the causes responsible for infant mortality and childhood morbidity, congenital anomalies make the major contribution, affecting $2-3 \%$ of all babies. ${ }^{1}$ They are composed of multiple malformations thought to be aetiologically and/or pathogenetically related. Syndromes that have cleft lip and/or cleft palate as one of the features are of interest in the search for aetiologic and genetic factors, and it is estimated that $30 \%$ of cleft cases are syndromic and about $70 \%$ are non-syndromic. ${ }^{1}$

Financial or Other, Competing Interest: None.

Submission 13-05-2015, Peer Review 24-06-2015,

Acceptance 01-07-2015, Published 11-11-2016.

Corresponding Author:

Dr. Nishar Ahamad M,

Haji Manzil, Post Mangilapadau,

Vittal-574243.

Dakshina Kannada

Karnataka.

E-mail: nissy925@yahoo.com

DOI: $10.14260 /$ jemds $/ 2016 / 1524$
Ocular manifestations such as refractive errors, strabismus, coloboma of the eyelids, ectropion and amblyopia are also quite common in these patients. ${ }^{2,3}$ This study was done to detect ocular involvement in patients with craniofacial anomalies and also to emphasise importance of routine ophthalmic examination in these patients.

\section{METHODOLOGY}

\section{Study Design}

This was a cross-sectional observational type of study. Study duration was 2 years dated from November 2012 to October 2014.

\section{Subjects}

All patients who presented to the Department of Ophthalmology of Justice KS Hegde Medical College Hospital, Mangalore with craniofacial anomalies were included in this study. All patients with CFA were included in the study irrespective of age of presentation, gender or history of previous corrective surgery. The patients were evaluated at the time of presenting to the Department of Ophthalmology of 
Justice KS Hegde Medical College, Mangalore. Duration of this study was dated from November 2012 to October 2014.

\section{PATIENT SELECTION CRITERIA Inclusion Criteria}

- Patients in any age group diagnosed with any Craniosynostosis or Clefting syndrome abnormalities.

\section{Exclusion Criteria}

- Patients with post-surgical (Enucleation, evisceration and exenteration, surgery involving the orbital walls) facial deformities and patients presenting with post-traumatic facial anomalies were excluded from this study.

\section{Sample Size}

The study was designed as a time bound study. Patients in any age group diagnosed with any Craniosynostosis or Clefting syndrome abnormalities presenting to the Department of Ophthalmology of Justice K. S. Hegde Medical College, Mangalore dated from November 2012 to October 2014 were examined.

\section{Data Collection}

The study was done after obtaining the approval from the Institutional Ethics Committee. Informed consent was taken from the patient or patient's caretaker, detailed ocular, medical, family history was obtained and recorded on a data sheet (Proforma).

Ocular examination included distant visual acuity assessment done using Snellen's chart or alternate testing methods were included when required which was appropriate for age of the patient. Paediatric visual acuity charts were used for children above 3 years of age, and for children below three years of age visual acuity testing was done by their ability to follow hand movements.

Ocular movements (Uniocular and binocular) were examined by asking patient to follow a moving target. Gaze abnormalities were detected, documented after evaluation by Hirschberg method, Krimsky's test and Prism bar cover test using horizontal or vertical prisms. Radiological investigation (X-ray or CT imaging) was done for the confirmation of hypertelorism if detected.

Slit lamp microscopy was done for evaluating anterior segment. Intraocular pressure was estimated in all the patients. For all patients above 8 years of age, Goldmann applanation tonometry and correction for central corneal thickness was done, Schiotz tonometry was done for all patients under the age of 8 years. For patients below the age of four years, IOP measurement was done under sedation in OT.

Dry refraction was done using a streak retinoscope for all patients above the age of 10 years. For patients below the age group of 10 years, cycloplegic refraction was done using cyclopentolate $1 \%$ eye drops. Patients those found to have refractive errors were instructed to come for a post-mydriatic test and during that presentation spectacles were prescribed.

All the patients in this study were subjected for fundus examination which was done by direct or indirect ophthalmoscopy using +20 D lens. In patients whose fundus could not be examined in the outpatient department, fundus was evaluated under general anaesthesia (Preoperatively if the patient was posted for corrective surgery) or under sedation. Preoperative radiological investigations (CT scan) were done in patients with craniosynostosis or clefting syndromes posted for corrective surgeries.

\section{Data Analysis}

This was an observational study, the data was collected and recorded on a data sheet (proforma). Data was later analysed and the percentage of ocular associations was calculated and was then compared with earlier studies done in which similar data analysis methods was used.

\section{RESULTS AND INTERPRETATION OF DATA}

- Total number of patients-151.

- Number of male patients-55.

- Number of female patients-96.

- History of consanguinity-14.

- Number of patients delivered at home-7.

- Number of patients with craniosynostosis-39.

- Crouzon syndrome-34.

- Apert syndrome-5.

- Number of patients with clefting syndromes-112.

- Goldenhar syndrome-15.

- Treacher Collins syndrome-18.

- Pierre Robin syndrome-24.

- Cleft lip and palate-55.

- Number of patients with eyelid abnormalities-33.

- Number of patients with refractive errors-57.

- Number of patients with abnormal gaze-33.

- Number of patients with abnormal fundus-4.

\section{RESULTS AND DISCUSSION}

Ocular Manifestations in Children with Craniofacial Anomalies are an important but easily overlooked part. This study was done to detect ocular anomalies in patients presenting with craniofacial anomalies and also to emphasise the importance of ophthalmic examination in these patients. In this study of ocular associations in patients with craniofacial anomalies, 151 patients with craniofacial anomalies referred to us from the craniofacial unit in our hospital were examined. Our study included 55 male patients and 96 female patients. The number of female patients was more in our study. But there is no gender predisposition ${ }^{4}$ for craniofacial anomalies. Majority of our patients hailed from a rural area which corresponds to data collected from various similar studies conducted in India. 4

Consanguineous marriage is a known risk factor ${ }^{5}$ for craniofacial anomalies and in our study out of 151 patients, 14 patients had positive history of consanguineous marriage. This indicates towards the importance of conducting health awareness programs and educating general population about harmful effects of consanguineous marriage especially in rural areas.

Two important categories 6 of craniofacial anomalies in which ocular involvement is common are Clefting syndromes and Craniosynostosis.

In our study which included 151 patients, 112 patients belonged to the clefting syndrome category and 39 patients to the craniosynostosis category.

In this study 15 patients were diagnosed to have Goldenhar syndrome. 12 patients had ocular involvement. Majority of the patients had refractive errors and limbal dermoids. 18 patients were diagnosed to have Treacher 
Collins syndrome out of which 12 had ocular involvement. 24 patients were diagnosed to have Pierre Robin syndrome out of which 6 had ocular involvement. 55 patients had cleft lip and palate out of which 3 had ocular involvement.

Goldenhar syndrome ${ }^{7}$ is a congenital defect characterised by incomplete development of the ear, nose, soft palate, lip, and mandible. Common clinical manifestations include limbal dermoids, preauricular skin tags, ptosis and strabismus. ${ }^{8}$

In our study, 34 patients had Crouzon's syndrome. Crouzon's syndrome 8 , 6, 9 is characterised by acrocephaly, exophthalmos, hypertelorism, strabismus, parrot-beaked nose, and hypoplastic maxilla. All patients in our study who were diagnosed to have Crouzon's syndrome had ocular involvement. Majority of the patients had gaze abnormalities (Exotropia10) and defective vision due to refractive errors. $18 \%$ of the patients in our study were amblyopic. This indicates towards the importance of ophthalmic examination in newborns with craniofacial anomalies as early as possible, even before corrective surgeries. In a retrospective study on patients with Crouzon's syndrome by Silva et al, ${ }^{9}$ visual impairment was found in at least 1 eye in $35 \%$ of patients and was bilateral in $9 \%$ of patients. The most common cause of visual impairment was amblyopia, which was present in $21 \%$ of patients, followed by optic atrophy in $7 \%$. In the same study, strabismus was also found in about $39 \%$ of patients.

Gaze abnormalities are common in craniofacial anomalies. Craniofacial anomalies are associated with an increased incidence of ocular deviations ${ }^{11}$ ranging between $40 \%$ to $60 \% .^{12,13}$ In a study done by Tan et al on 40 patients with craniosynostosis, $60 \%$ of the patients in that study group were found to have ocular deviations.

In our study, we found out that $77.48 \%$ of the patients were orthophoric and $22.52 \%$ of the patients had abnormal gaze. Craniofacial anomalies are associated with an increased incidence of ocular deviations 11 ranging between $40 \%$ to $60 \% .^{12,13}$ In a study done by Tan et $\mathrm{al}^{14}$ on 40 patients with craniosynostosis, $60 \%$ of the patients in that study group were found to have ocular deviations. In that study exotropia was more common than esodeviations, though various other studies have shown the incidence of esodeviations compared to exodeviations to be roughly equal.15,14 In our study, 5.29\% of the patients with gaze abnormalities had esotropia and $16.55 \%$ of the patients had exotropia. $11.26 \%$ of orthophoric gaze patients had nystagmus.

In our study, we found out majority of the patients with craniofacial anomalies had defective vision. Cause for defective vision in patients with craniofacial anomalies could be due to refractive errors, amblyopia which could be due to uncorrected refractive errors or strabismus, anisometropia or due to cataract. Diminution of visual acuity was the most common ocular association in our study. Diminished visual acuity in craniofacial anomalies could be due to refractive error, or as a result of amblyopia due to uncorrected refractive errors, anisometropia, strabismus or cataract. Dysmorphism affecting size or shape of the globe ${ }^{15}$ explains the reason for higher incidence of refractive errors in patients with craniofacial anomalies compared to general population. In a study $^{9}$ with a sample size of 131 patients with Crouzon Syndrome by Silva et al, $35 \%$ of the patients were found to have a visual impairment in at least one eye. In a similar study on Apert syndrome by Khong et al,16 $54 \%$ of the 61 patients were found to have visual impairment in at least one eye.
In our study out of 151 patients, $51.99 \%$ of the patients were emmetropic, $22.51 \%$ hypermetropic, $5.29 \%$ hypermetropic astigmatism, 6\% myopia, 5\% myopic astigmatism. Vision could not be assessed in 15 patients. Hypermetropia was the commonest refractive error detected.

Craniofacial anomalies are known to cause anomalies of the skull and face and parents are usually concerned more about the appearance of these patients and hence ignore the ocular anomalies which can lead to permanent defects like defective vision. Uncorrected refractive errors can lead to amblyopia, but early detection and correction of these refractive errors can prevent amblyopia. In our study, we found out that $18 \%$ of the patients were amblyopic. Studies have shown an incidence of amblyopia ${ }^{9,17}$ between ranges of $20 \%$ to $35 \%$ of all patients. This clearly indicates towards the importance of ophthalmic examination as early as possible in patients with craniofacial anomalies in order to prevent permanent damage of eye.

Craniofacial abnormalities can manifest with vast variety of anterior segment abnormalities that include eyelid abnormalities such as ptosis and coloboma, ectropion and epicanthus inversus. ${ }^{8}$ Other abnormalities include limbal dermoid, cataract or iris coloboma. In a study ${ }^{11}$ by Shah et al to determine the structural abnormalities in patients with anophthalmos, coloboma and microphthalmos, 32.1\% of 135 patients were found to have a craniofacial anomaly. In our study, we found out 14 patients had ptosis. 2 patients had epicanthus inversus, 5 patients with ectropion, 4 patients with entropion, 5 patients had lid coloboma, 3 had symblepharon. Ptosis was the commonest lid anomaly in our study. Other abnormalities include limbal dermoids which were found in 9 patients and hypertelorism in 14 patients.

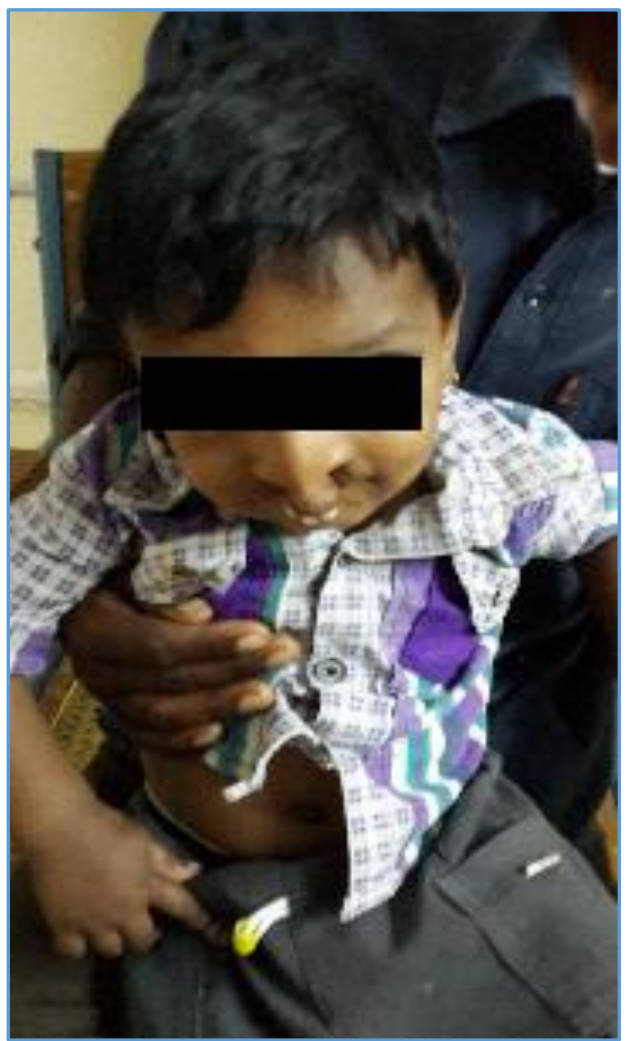

Figure 1. Patient with Clefting Syndrome 


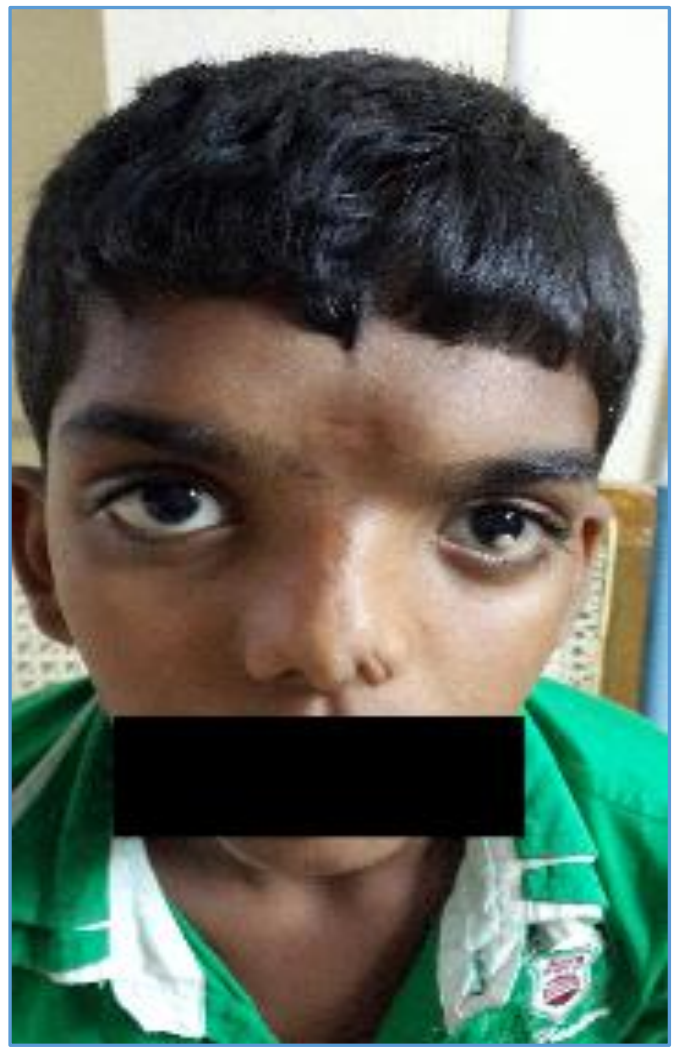

Figure 2. Patient with Limbal Dermoid

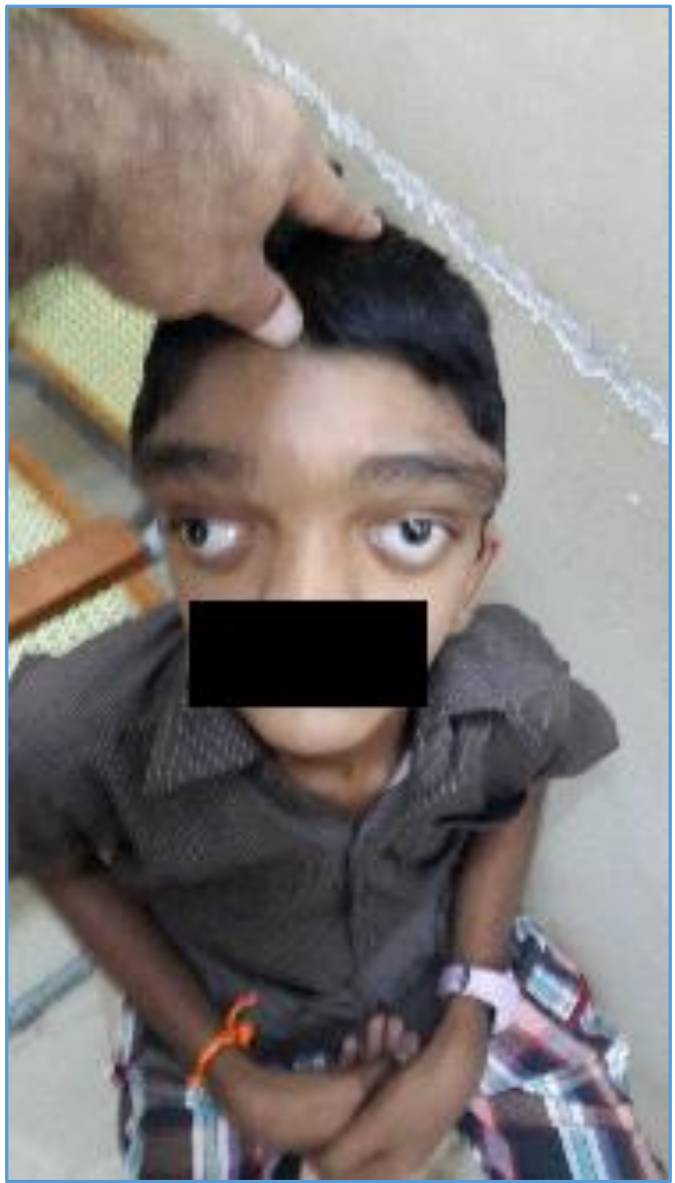

Figure 3. Patient with Hypertelorism and Right Eye Exotropia

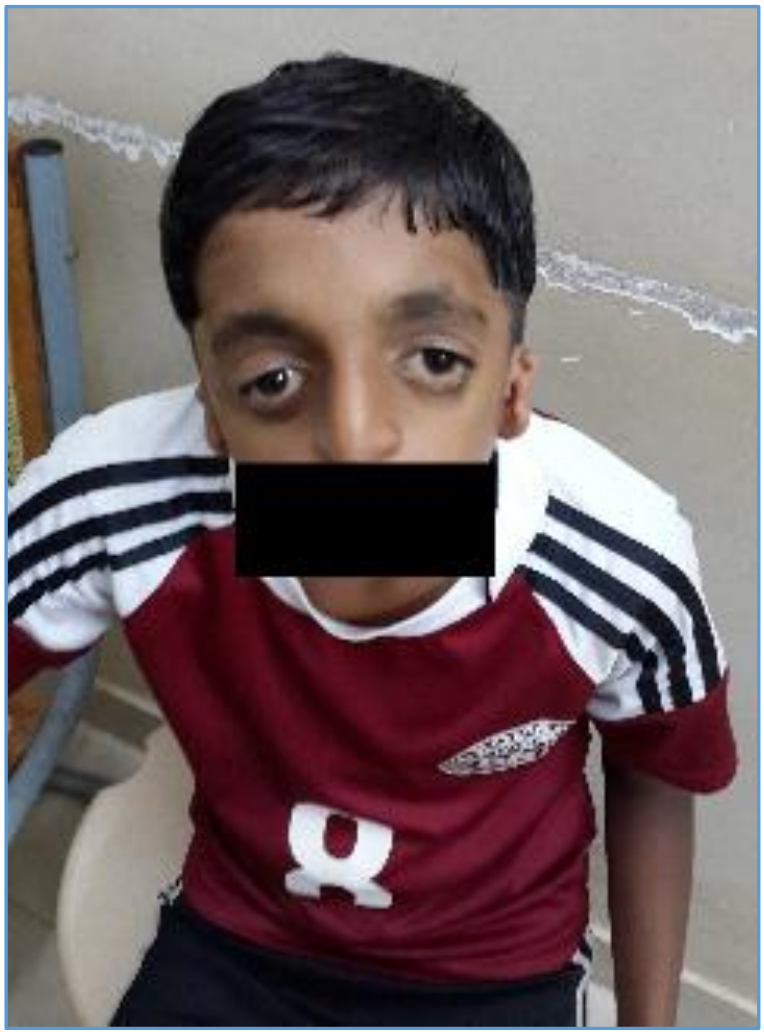

Figure 4. Patient with Treacher Collins syndrome

\section{Limitations of the Study and Suggestions}

1. This being a time-bound study, patient sample size in this study was small and a larger sample size might have given a broader understanding of the ocular associations in patients with craniofacial anomalies.

2. Accurate visual acuity evaluation could not be done in the 0-3 age group of patients in our study. It had to be done by assessing their ability to follow moving object or light source. More accurate assessment of visual acuity using an optokinetic drum or other methods would have yielded better results.

\section{CONCLUSION}

Ocular manifestations in Children with Craniofacial Anomalies are an important but easily overlooked part as the patient and his family will be more focused about the cosmetic disfigurement caused by this condition.

Majority of the patients in our study had refractive errors which only requires ophthalmic evaluation in outpatient department and spectacle correction for the refractive errors. Ophthalmic examination as early as possible in patients with craniofacial anomalies will help in detection of gaze abnormalities and lid abnormalities, surgical management of these anomalies will prevent permanent ocular damage. Our study attempts to highlight the need for a comprehensive ophthalmic evaluation in all patients who are diagnosed to have craniofacial anomalies.

Findings in our study suggest that patients with craniofacial anomalies can have variety of ocular associations which indicate the importance of routine ophthalmic evaluation in all patients who are diagnosed to have craniofacial anomalies. Early diagnosis of a simple refractive error in these patients can prevent amblyopia which would greatly benefit the patient. 


\section{REFERENCES}

1. Mossey PA, Castilla EE. Global registry and database on craniofacial anomalies. In: WHO registry meeting on craniofacial anomalies (2001: Bauru, Brazil) Geneva: WHO 2003:1-51.

2. Skuta GL, Cantor LB, Weiss JS. Ocular development. In: Section 2 basic and clinical science course. USA: AAO 2008. P. 139.

3. Cook CS, Sulik KK, Wright KW. Embryology. In: Wright KW, Spiegel PH, Thompson LS. Handbook of Pediatric NeuroOphthalmology. $2^{\text {nd }}$ edn. USA: Springer Science+Business Media 2006. P. 34.

4. Cohen MM, Kreiborg S. Birth prevalence studies of the Crouzon syndrome-comparison of direct and indirect methods. Clin Genet 1992;41(1)12-5.

5. Abdulrazzaq YM, Bener A, al-Gazali LI, et al. A study of possible deleterious effects of consanguinity. Clin Genet 1997;51(3):167-73.

6. Fries PD, Katowitz JA. Congenital craniofacial anomalies of ophthalmic importance. Surv Ophthalmol 1990:35(2): 87-119.

7. Forbes BJ. Congenital Craniofacial anomalies. Current Opinion Ophthalmol 2010;21(5):367-74.

8. Greaves B, Walker J, Wybar K. Disorders of ocular motility in craniofacial dysostosis. J R Soc Med 1979;72(1):21-4.

9. Gray TL, Casey T, Selva D, et al. ophthalmic sequelae of Crouzon syndrome. Ophthalmology 2005;112(6):1129-34.
10. Poswillo D. The aetiology and pathogenesis of craniofacial deformity. Development 1988;(103 Suppl):207-12.

11. Shah SP, Taylor AE, Sowden JC, et al. Anophthalmos, microphthalmos and Coloboma in the United Kingdom: clinical features, results of investigations and early management. Ophthalmology 2012;119(2):362-8.

12. Khan SH, Nischal KK, Dean F, et al. Visual outcomes and amblyogenic risk factors in craniosynostotic syndromes: a review of 141 cases. Br J Ophthalmol 2003;87(8):9991003.

13. MacDonald IM, Tran M, Musarella MA. Ocular genetics: current understanding. Surv Ophthalmol 2004;49(2): 15996.

14. Tan KP, Sargent MA, Poskitt KJ, et al. Ocular over elevation in adduction in craniosynostosis: is it the result of excyclorotation of the extraocular muscles? J AAPOS 2005;9(6):550-7.

15. Hertle RW, Ziylan S, Katowitz JA. Ophthalmic features and visual prognosis in the Treacher-Collins syndrome. Br J Ophthalmol1993;77(10):642-5.

16. Khong JJ, Anderson P, Gray TL, et al. Ophthalmic Findings in Apert's syndrome after craniofacial surgery. Twentynine years' experience. Ophthalmology 2006;113(2):34752.

17. Kolk VCA, Beaty T. Etiopathogenesis of Craniofacial anomalies. Clin Plast Surg 1994;21(4):481-8. 